\title{
Greedy kernel methods for accelerating implicit integrators for parametric ODEs
}

\author{
T. Brünnette ${ }^{1}$, G. Santin ${ }^{* 1}$, and B. Haasdonk ${ }^{\dagger 1}$ \\ ${ }^{1}$ Institute for Applied Analysis and Numerical Simulation, University \\ of Stuttgart, Germany
}

August 30, 2018

\begin{abstract}
We present a novel acceleration method for the solution of parametric ODEs by single-step implicit solvers by means of greedy kernelbased surrogate models. In an offline phase, a set of trajectories is precomputed with a high-accuracy ODE solver for a selected set of parameter samples, and used to train a kernel model which predicts the next point in the trajectory as a function of the last one. This model is cheap to evaluate, and it is used in an online phase for new parameter samples to provide a good initialization point for the nonlinear solver of the implicit integrator. The accuracy of the surrogate reflects into a reduction of the number of iterations until convergence of the solver, thus providing an overall speedup of the full simulation. Interestingly, in addition to providing an acceleration, the accuracy of the solution is maintained, since the ODE solver is still used to guarantee the required precision. Although the method can be applied to a large variety of solvers and different ODEs, we will present in details its use with the Implicit Euler method for the solution of the Burgers equation, which results to be a meaningful test case to demonstrate the method's features.
\end{abstract}

\section{Problem setting}

We consider a $d$-dimensional, autonomous, first order parametric initial value problem: For a given vector of parameters $\mu \in \mathcal{P} \subset \mathbb{R}^{p}$ from an admissible set $\mathcal{P}$, solve

\footnotetext{
*santinge@mathematik.uni-stuttgart.de, orcid.org/0000-0001-6959-1070

†haasdonk@mathematik.uni-stuttgart.de
} 


$$
\operatorname{IVP}(\mu):\left\{\begin{aligned}
\dot{u}(t, \mu) & =f(u(t, \mu), \mu), t \in[0, T] \\
u(0) & =u_{0}(\mu) \in \mathbb{R}^{d} .
\end{aligned}\right.
$$

We assume that $\operatorname{IVP}(\mu)$ has a unique solution $u(t, \mu):=u\left(t, \mu, u_{0}(\mu)\right)$, $t \in[0, T]$, for any value $\mu \in \mathcal{P}$ and for any initial value $u_{0}(\mu) \in \mathbb{R}^{d}$. Conditions on $f$ such that this requirement is fulfilled are well known, and we refer e.g. to [5] for details.

Existence and uniqueness of solutions allow to define a parametric time evolution or flow mapping

$$
\Phi\left(t, u_{0}(\mu)\right):=u(t, \mu),
$$

which maps the initial value and the time to the corresponding solution vector in $\mathbb{R}^{d}$, and for which it holds $\Phi(s, u(t, \mu))=u(t+s, \mu)$. Although the dependency on the parameters in $\operatorname{IVP}(\mu)$ can be quite general, we require that $\Phi\left(t, u_{0}(\mu)\right) \neq \Phi\left(s, u_{0}(\nu)\right)$ for all $t, s \in[0, T]$ and for all $\mu, \nu \in \mathcal{P}$ s.t. $(t, \mu) \neq(s, \nu)$, i.e., different parameters lead to non intersecting trajectories.

We further assume to have an implicit time integrator which is able to numerically solve $\operatorname{IVP}(\mu)$ with any given accuracy, provided a small enough time step is used. Although our acceleration algorithm applies to general single-step integration methods, in this paper, for the sake of presentation, we will concentrate on the Implicit Euler method (IE), and we refer again to [5] for details on its accuracy.

Such integration method considers a timestep $\Delta t>0$ and a uniform time discretization of $[0, T]$ in $N_{t}:=N_{\Delta t} \in \mathbb{N}$ intervals $0=t_{0}<t_{1}<\cdots<$ $t_{N_{t}} \leq T$, with $t_{i+1}-t_{i}=\Delta t, 0 \leq i \leq N_{t}-1$, and computes a discrete-time approximation of $u$ as $u_{i}(\mu) \approx u\left(t_{i}, \mu\right), 0 \leq i \leq N_{t}$.

A numerical time evolution map $\phi: \mathbb{R} \times \mathbb{R}^{d} \rightarrow \mathbb{R}^{d}$ analogous to (1) can be defined from the approximate solution as

$$
\phi\left(\Delta t, u_{i}(\mu)\right):=u_{i+1}(\mu), 0 \leq i \leq N_{t}-1,
$$

i.e., the solution vector at the current time point is mapped to the solution vector at the next time point. Observe that, under the hypotheses of arbitrary accuracy of the integration method and of non intersection of the trajectories, we assume that also the discrete trajectories are non intersecting. This means that $\phi$ is a globally defined function independent of the parameter $\mu \in \mathcal{P}$.

At each discrete time point, the integrator needs to solve a generally nonlinear, $d$-dimensional system of equations to determine the approximation $u_{i}(\mu)$. We assume that this equation is solved with an iterative method, e.g., the Newton method, using an initialization $\bar{u}_{i}(\mu) \in \mathbb{R}^{d}$ at time $t_{i}$. Common choices of this value for the IE method are, e.g., the previous approximation $u_{i-1}(\mu)$ or the approximation obtained by one step of the Explicit Euler method. 
The goal of this paper is to present a way to accelerate the computation of the numerical solution $\left\{u_{i}(\mu)\right\}_{i}$ for an arbitrary parameter vector $\mu \in \mathcal{P}$.

The acceleration is realized by constructing a surrogate $s_{\phi}: \mathbb{R} \times \mathbb{R}^{d} \rightarrow \mathbb{R}^{d}$ of the numerical time evolution map $\phi$ such that $s_{\phi}(\Delta t, u) \approx \phi(\Delta t, u)$ for all $(\Delta t, u) \in[0, T] \times \mathbb{R}^{d}$, while the evaluation of $s_{\phi}$ is much faster than the evaluation of $\phi$. This surrogate is computed in an offline phase in a datadependent fashion, i.e., it is trained using a set of precomputed numerical trajectories $\left\{u_{i}\left(\mu_{j}\right)\right\}_{i j}$ for multiple parameter values $\mathcal{P}_{t r}:=\left\{\mu_{1}, \ldots, \mu_{N_{\mu}}\right\} \subset$ $\mathcal{P}, N_{\mu} \in \mathbb{N}$ and possibly multiple timesteps $\Delta t$.

In the online phase, for a new parameter $\mu \in \mathcal{P}$ the numerical solution is computed by the same time integrator and with timestep $\Delta t$, and, at each time $t_{i}$, the nonlinear solver is initialized by $s_{\phi}\left(\Delta t, u_{i-1}\right)$, i.e., $\bar{u}_{i}(\mu) \in \mathbb{R}^{d}$ is replaced by the surrogate prediction based on the previous timestep.

If the surrogate is accurate, $s_{\phi}\left(\Delta t, u_{i-1}(\mu)\right)$ is a good approximation of $\phi\left(\Delta t, u_{i-1}(\mu)\right)=u_{i}(\mu)$, so the nonlinear solver will converge in possibly significantly less iterations, ideally in 0 iterations if a residual criterion is used before starting the fix-point loop. This reduction of the iterations, combined with the fast evaluation of $s_{\phi}$, will produce a speedup of the overall computational time. Moreover, since the same time integrator and nonlinear solver are used in the accelerated algorithm, we should expect no degradation of the accuracy, provided the surrogate prediction is accurate enough so that the initialization point is within the area of convergence of the nonlinear solver. This is in contrast to general surrogate modeling or model reduction, where the approximation typically results in an accuracy loss.

The surrogate is constructed using the Vectorial Kernel Orthogonal Greedy Algorithm (VKOGA) [11, which will be discussed in Section 2, In particular, it is a kernel-based interpolation algorithm that constructs a nonlinear surrogate $s_{\phi}$. The full specification of the training data and the complete acceleration algorithm will be described in Section 3, but we anticipate that arbitrary unstructured trajectory data $\left\{u_{i}\left(\mu_{j}\right)\right\}_{i j}$ in possibly high dimension $d$ can be used. We will conclude this paper with different numerical experiments in Section 4 to demonstrate the capabilities of our method.

Moreover, similar acceleration methods have been presented in the papers 1, 2, where instead a linear surrogate is employed.

\section{Kernel based surrogates and the VKOGA}

We briefly outline here the fundamentals of interpolation with kernels and of the VKOGA algorithm, and we refer to [10] and to [11, 4] for the respective details.

We assume to have a function $f: \Omega \subset \mathbb{R}^{p} \rightarrow \mathbb{R}^{q}$ and a training dataset composed of pairwise distinct data points $X:=\left\{x_{i}\right\}_{i=1}^{N} \subset \Omega$ and data values 
$Y:=\left\{f\left(x_{i}\right)\right\}_{i=1}^{N} \subset \mathbb{R}^{q}$. We will specify in the following section the definition of the dataset for the current algorithm.

The general form of the surrogate is

$$
s_{f}(x):=\sum_{j=1}^{N} \alpha_{j} K\left(x, x_{j}\right), x \in \Omega,
$$

where $\alpha_{j} \in \mathbb{R}^{q}$ are coefficient vectors and $K: \Omega \times \Omega \rightarrow \mathbb{R}$ is a symmetric and strictly positive definite kernel. This means that the matrix $A_{X, K} \in \mathbb{R}^{N \times N}$, $\left(A_{X, K}\right)_{i j}:=K\left(x_{i}, x_{j}\right)$ is positive definite for all $N \in \mathbb{N}$ and for all sets $X \subset \Omega$ of $N$ pairwise distinct points. A particular $K$, i.e., the Gaussian kernel $K(x, y):=\exp \left(-\varepsilon^{2}\|x-y\|_{2}^{2}\right)$, with a positive shape parameter $\varepsilon>0$, will be used in Section 4 .

The coefficient vectors in (3) can be uniquely determined by imposing interpolation conditions

$$
s_{f}\left(x_{i}\right):=f\left(x_{i}\right), \quad 1 \leq i \leq N,
$$

which result, defining $\alpha^{T}:=\left[\alpha_{1}, \ldots, \alpha_{N}\right], b^{T}:=\left[f\left(x_{1}\right), \ldots, f\left(x_{N}\right)\right], \alpha, b \in$ $\mathbb{R}^{q \times N}$, in the solution of the linear system $A_{X, K} \alpha=b$. This, indeed, has a unique solution as $A_{X, K}$ is positive definite by assumption.

This interpolation method is well studied, and we just recall that convergence rates are proven for functions $f$ in the space $\mathcal{H}_{K}(\Omega)$, which is a Reproducing Kernel Hilbert Space (RKHS) associated to the particular kernel $K$, and which is norm equivalent to a Sobolev space $W_{2}^{\tau}(\Omega), \tau>d / 2$, for certain kernels (see [10]).

The goal of the VKOGA is to approximate the surrogate (3) by a sparse expansion of the same form, i.e., one where most of the $\alpha_{j}$ are the zero vector. A good selection of the sparsity pattern results into an approximate surrogate which is as good as the full one, while being much faster to evaluate, since the sum involves only $n \ll N$ elements. The selection of the nonzero coefficients and their computation is realized by a greedy procedure in $\mathcal{H}_{K}(\Omega)$, which iteratively selects nested data point sets $\emptyset \subset X_{n-1} \subset X_{n} \subset \Omega$ by maximizing a selection criterion at each step, and solves the corresponding interpolation problem. Possible choices in the VKOGA are the $f_{-}, P$ , and $f / P$-greedy selection rules [9, 3, 7]. The algorithm has theoretical grounds, e.g. provable convergence rates [7, 11, which are also quasi-optimal in Sobolev spaces for $P$-greedy [8, and has been successfully applied in several application contexts, e.g. 6]. Moreover, the numerical computation of the surrogate can be efficiently implemented using a partial Cholesky decomposition of the kernel matrix $A_{X, K}$, where only the columns appearing in the sparse surrogate need to be computed and stored. 


\section{The complete algorithm: VKOGA-IE}

We can now describe the complete algorithm, which we name VKOGA-IE. The target function is $f:=\phi$, which is defined on $\Omega:=[0, T] \times \mathbb{R}^{d}$ to $\mathbb{R}^{d}$, i.e., $p:=d+1, q:=d$. What remains to specify is the exact definition of the training set $(X, Y)$ used by VKOGA to construct the surrogate $s_{\phi}$, as described in the previous section. As mentioned in Section 1, we solve $\operatorname{IVP}(\mu)$ for $N_{\mu} \in \mathbb{N}$ different parameters from a parameter-training set $\mathcal{P}_{t r} \subset \mathcal{P}$, each $\mu_{j}$ with a timestep $\Delta t_{j}$. If the same parameter is used more than once with different timesteps, we just count it multiple times in $\mathcal{P}_{t r}$. This generates trajectory data which we assign at temporary sets $X_{j}:=\left\{\left(\Delta t_{j}, u_{i}\left(\mu_{j}\right)\right)\right\}_{i=0}^{N_{t}-1}, Y_{j}:=\left\{u_{i+1}\left(\mu_{j}\right)\right\}_{i=0}^{N_{t}-1}$, representing input-output pairs of $\phi$. The dataset is defined as $X:=\cup_{j=1}^{N_{\mu}} X_{j}, Y:=\cup_{j=1}^{N_{\mu}} Y_{j}$. The complete offline phase is summarized in Algorithm [1.

Instead of working with a fixed kernel shape parameter $\varepsilon>0$, typically step 13 implies a parameter selection procedure, e.g. via cross validation. Moreover, we assume for simplicity that $T / \Delta t \in \mathbb{N}$. In the online phase,

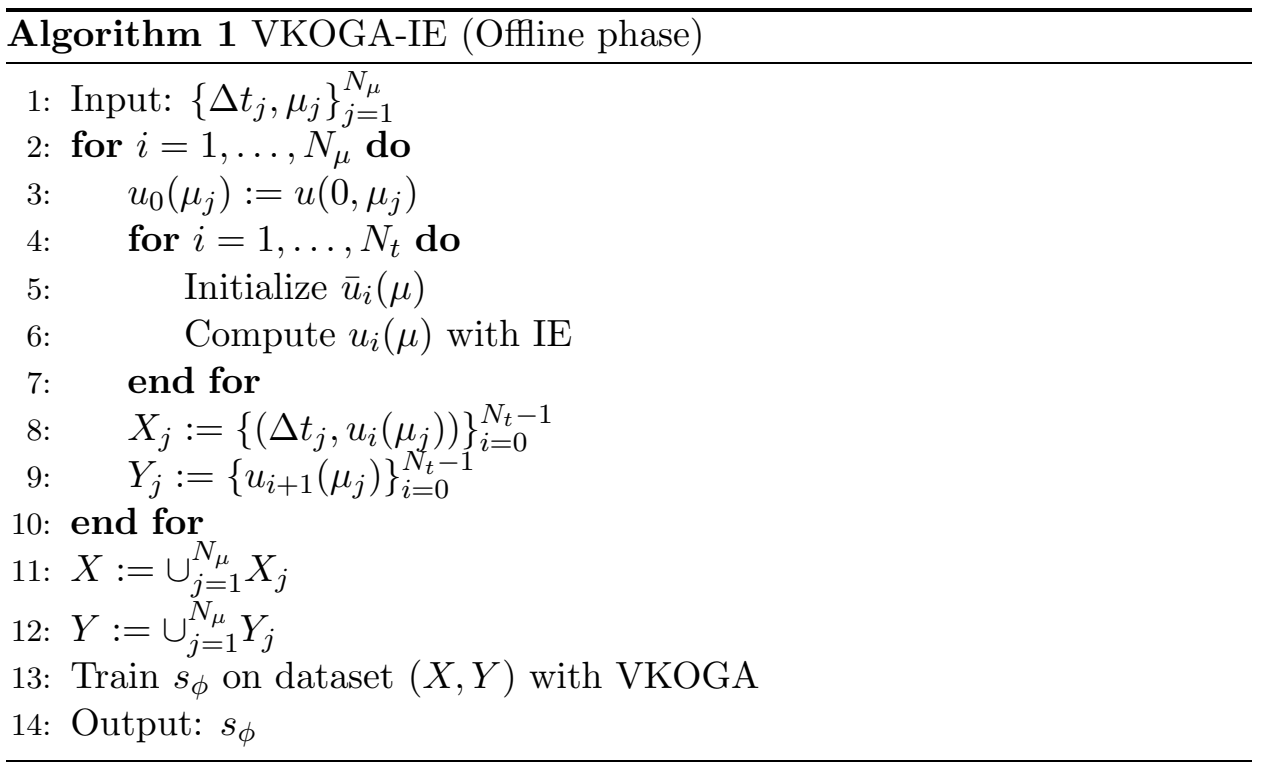

instead, we only need to run the IE method and solve at each iteration the nonlinear equation using the initialization provided by the surrogate, as described in Algorithm 2 , 


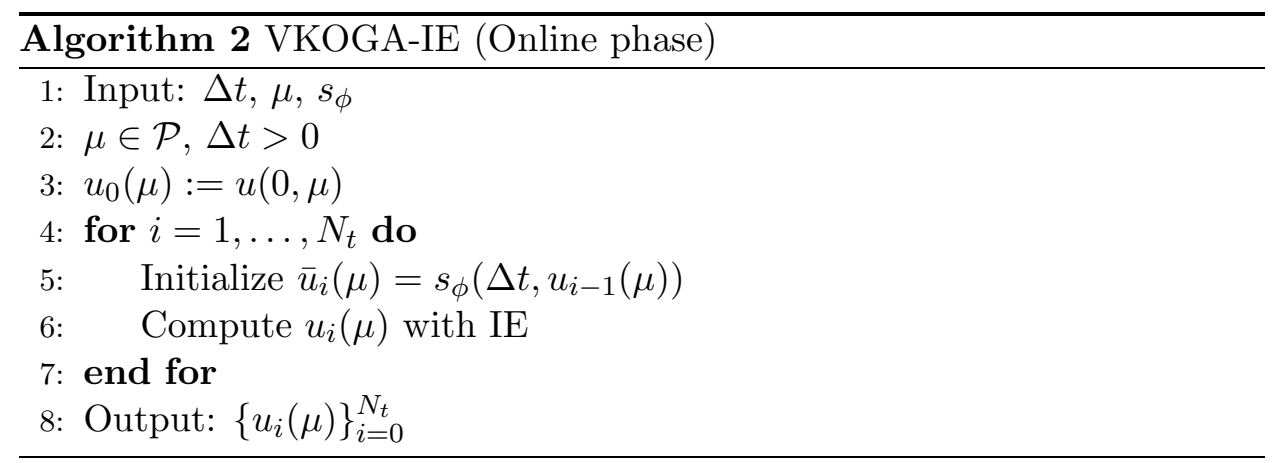

\section{Experiments}

To demonstrate the features of VKOGA-IE, we consider the Burgers equation

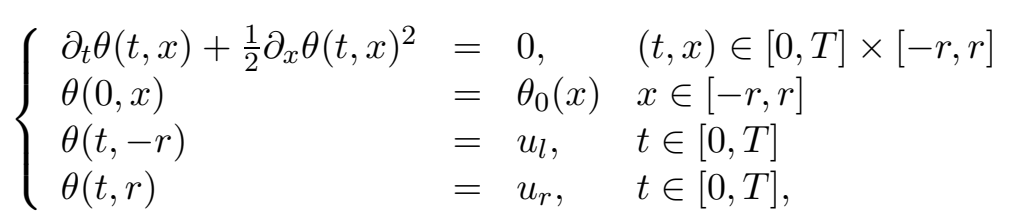

which is transformed into an ODE by a semi-discrete finite volume discretization in space based on the Lax-Friedrichs flux. We consider $d:=200$ cells in $(-r, r)$ with $r:=5$, and $T$ as specified later. This produces a $d$ dimensional $\operatorname{IVP}(\mu)$ depending on a two-dimensional parameter vector $\mu:=\left(u_{l}, u_{r}\right)$. We concentrate here on shock wave solutions, i.e., $u_{l}>u_{r}$. The resulting ODE is then simulated from $t=0$ to $t=T$, with varying time-step $\Delta t$. The nonlinear system is solved at each timestep using the Newton method, which is terminated with a maximal number of 100 iterations or when a tolerance of $10^{-14}$ on the residual is reached. To have more training points, all the training sets in the following are generated with training time $T=T_{t r}:=4$.

The VKOGA is run with the Gaussian kernel and with a termination tolerance of $10^{-12}$. The kernel depends on a parameter $\varepsilon>0$, which is chosen via 5 -fold cross validation from a set of 50 logarithmically equally spaced values in $\left[10^{-4}, 10^{2}\right]$.

The first experiment uses a fixed $\Delta t=0.01$ and a single training parameter $\mathcal{P}_{t r}=\{(3.4,0.2)\}$, i.e., $N=400=T / \Delta t$. Observe that a fixed $\Delta t$ means that the model is in practice $d$ to $d$ dimensional. The VKOGA selects $n=67$ points, and the model is tested to solve $\operatorname{IVP}(\mu)$ with parameters $\mathcal{P}_{t e}:=\{(3.4+i 0.2,0.2+j 0.2), i, j \in\{-1,0,1\}\}$ and $T=T_{t e}:=2$. The results are summarized in Table 1, The average number of iterations for the standard initialization with the previous value ('Old value' column) and with the VKOGA model ('VKOGA' column) are reported, as well as the test parameters where the minimal and maximal gain of our technique is realized. The table contains also the computational times in seconds, which 
are the averages over 10 repetitions of the same simulation based on a Matlab implementation. It is evident that a good speedup is reached when the model is tested on the training parameter, while the quality degrades for different ones.

\begin{tabular}{||c||c|c||c|c||c|c||c||}
\hline \multicolumn{1}{||c||}{} & \multicolumn{2}{c||}{ Old value } & \multicolumn{2}{c||}{ VKOGA } & \multicolumn{2}{c||}{ Gain } & \\
\hline & iter & time & iter & time & iter & time & $\mu$ \\
\hline Mean & 25.40 & 1.38 & 25.83 & 1.50 & $-1.70 \%$ & $-8.55 \%$ & \\
\hline Min & 24.27 & 1.29 & 27.84 & 1.63 & $-14.69 \%$ & $-25.97 \%$ & $(3.2,0.4)$ \\
\hline Max & 25.30 & 1.34 & 16.41 & 0.98 & $35.11 \%$ & $26.59 \%$ & $(3.4,0.2)$ \\
\hline
\end{tabular}

Table 1: Results of the first experiment with $\mathcal{P}_{t r}=\{(3.4,0.2)\}$ and fixed timestep $\Delta t=0.01$.

The second experiment uses a model trained again with fixed $\Delta t=0.01$ and the same test parameters $\mathcal{P}_{t e}$, but instead with training parameters $\mathcal{P}_{t r}:=\{(3.2,0),(3.2,0.4),(3.6,0),(3.6,0.4)\}$, i.e., the corners of the square containing $\mathcal{P}_{t e}$. The resulting training set has $N=400 \times 4=1600$ points, and the VKOGA selects $n=219$ points. The results are summarized in Table 2. In this case, as expected, we obtain a significant reduction of the number of iterations for all the test parameters. The minimal reduction is realized for the parameter $\mu=(3.2,0.2)$, which is the farthest from the training set. This is a further indication that the quality of the model degrades with the distance from the training set, which is a reasonable behavior but also a promising feature, since a model trained on a larger parameter training set should improve the acceleration. This reduction is reflected also in a speedup in terms of computational cost, except in one case reported in the table. This suggests that the additional cost required by the evaluation of the kernel model is relevant in the case of a small reduction of the number of iterations. Nevertheless, the computational time is highly dependent on the implementation, while the number of iterations is not.

\begin{tabular}{||c||c|c||c|c||c|c||c||}
\hline \multicolumn{1}{||c||}{} & \multicolumn{2}{c||}{ Old value } & \multicolumn{2}{c||}{ VKOGA } & \multicolumn{2}{c||}{ Gain } & \\
\hline & iter & time & iter & time & iter & time & $\mu$ \\
\hline Mean & 25.40 & 1.36 & 20.31 & 1.17 & $19.98 \%$ & $13.35 \%$ & \\
\hline Min & 25.29 & 1.28 & 24.25 & 1.33 & $4.13 \%$ & $-4.18 \%$ & $(3.4,0.2)$ \\
\hline Max & 25.12 & 1.36 & 16.68 & 0.97 & $33.60 \%$ & $28.21 \%$ & $(3.2,0)$ \\
\hline
\end{tabular}

Table 2: Results of the second experiment, i.e., model trained with $\mathcal{P}_{t r}:=$ $\{(3.2,0),(3.2,0.4),(3.6,0),(3.6,0.4)\}$ and fixed timestep $\Delta t=0.01$.

Finally, we test the behavior of the method with respect to a change in the timestep $\Delta t$. To this end, we use $\mathcal{P}_{t r}=\mathcal{P}_{t e}=\{(3.4,0.2)\}$, but we train the model with the solutions computed for $\Delta t \in\{0.01,0.005,0.001\}$ and test for 10 logarithmically equally spaced timesteps in $[0.001,0.05]$. The 
results are reported in Table 3. Also in this case we achieve a reduction of the number of Newton iterations in all cases, even if this reduction is not sufficient in the case of the smallest timestep to achieve a computational speedup, since the number of iterations is already quite small. Nevertheless, the reduction of the number of iterations suggests that the kernel model captures well the dependence on the timestep, so one could expect to use this technique in more general settings without the need of including in the training sets many solutions obtained with different timesteps.

\begin{tabular}{||c||c|c||c|c||c|c||c||}
\hline \multicolumn{1}{||c||}{} & \multicolumn{1}{c||}{ Old value } & \multicolumn{2}{c||}{ VKOGA } & \multicolumn{2}{c||}{ Gain } & \\
\hline & iter & time & iter & time & iter & time & $\Delta t$ \\
\hline Mean & 32.45 & 2.28 & 29.20 & 2.29 & $11.29 \%$ & $4.22 \%$ & \\
\hline Min & 9.20 & 5.74 & 8.98 & 6.46 & $2.45 \%$ & $-12.44 \%$ & $10^{-3}$ \\
\hline Max & 23.98 & 1.07 & 19.21 & 0.94 & $19.88 \%$ & $12.51 \%$ & $8.7910^{-3}$ \\
\hline
\end{tabular}

Table 3: Results of the third experiment, i.e., $\mathcal{P}_{t r}=\{(3.4,0.2)\}$ and multiple timesteps.

\section{Conclusion and further work}

In this work we described a general nonlinear forecasting method used for the acceleration of implicit ODE integrators. The method is suited for parametric problems and multi-query scenarios, and it realizes a significant acceleration possibly without accuracy degradation.

The algorithm can be extended to non-autonomous ODEs, adaptivetimestep or multi-stage Runge-Kutta time integrators. In each case, more simulation data should be included in the training set, such as the current time or the partial solutions of the intermediate stages.

Another interesting aspect that could be investigated is the analysis of the accuracy of the method. Indeed, if it is possible to prove that the surrogate has a small enough uniform error, it would be guaranteed that the initialization point is inside the convergence area of the nonlinear solver.

\section{Acknowledgments}

The authors would like to thank the German Research Foundation (DFG) for financial support of the project within the Cluster of Excellence in Simulation Technology (EXC 310/2) at the University of Stuttgart.

\section{References}

[1] K. Carlberg, L. Brencher, B. Haasdonk, and A. Barth, Datadriven time parallelism via forecasting, submitted to SIAM J. of Sci. Comp., 2016. 
[2] K. Carlberg, J. Ray, and B. van Bloemen WaAnders, Decreasing the temporal complexity for nonlinear, implicit reduced-order models by forecasting, Computer Methods in Applied Mechanics and Engineering 289 (2015), 79 - 103.

[3] S. De Marchi, R. Schaback, And H. Wendland, Near-optimal data-independent point locations for radial basis function interpolation, Adv. Comput. Math. 23:3 (2005), 317-330.

[4] B. HaAsdonk and G. Santin, Greedy kernel approximation for sparse surrogate modelling, Proceedings of the KoMSO Challenge Workshop on Reduced-Order Modeling for Simulation and Optimization, 2017.

[5] E. Hairer, S. P. Nø RSett, and G. Wanner, Solving Ordinary Differential Equations. I: Nonstiff problems., second ed., Springer Series in Computational Mathematics, vol. 8, Springer-Verlag, Berlin, 1993,

[6] T. Köppl, G. Santin, B. Haasdonk, and R. Helmig, Numerical modelling of a peripheral arterial stenosis using dimensionally reduced models and machine learning techniques, Tech. report, University of Stuttgart, 2017.

[7] S. Müller And R. Schaback, A Newton basis for kernel spaces, J. Approx. Theory 161:2 (2009), 645-655.

[8] G. SAntin AND B. HAASDOnK, Convergence rate of the data-independent P-greedy algorithm in kernel-based approximation, Dolomites Res. Notes Approx. 10 (2017), 68-78.

[9] R. Schaback and H. Wendland, Adaptive greedy techniques for approximate solution of large RBF systems, Numer. Algorithms 24:3 (2000), 239-254.

[10] H. Wendland, Scattered Data Approximation, Cambridge Monographs on Applied and Computational Mathematics, vol. 17, Cambridge University Press, Cambridge, 2005.

[11] D. Wirtz And B. HaAsdonk, A vectorial kernel orthogonal greedy algorithm, Dolomites Res. Notes Approx. 6 (2013), 83-100. 